\title{
Phantom Pain in Major Lower Limb Amputation: Primary Ligation Cauterization vs Traction Neurectomy
}

\author{
Ahmad Redzuan Arshad ${ }^{1}$, Nazri Mohd Yusoff ${ }^{1}$ \\ ${ }^{1}$ Department of Orthopaedics, Traumatology and Rehabilitation, Kulliyyah of \\ Medicine, International Islamic University Malaysia
}

Presenter: Ahmad Redzuan Arshad

Introduction: Phantom Limb Pain (PLP) is haunting amputees in silent. Neuroma hyperexcitability is one of the popular peripheral theories which can be addressed during primary amputation by orthopaedic surgeons. There was no study comparing type of neurectomy association with PLP. The objective was to compare the occurrence of PLP between conventional and alternative techniques of neurectomy in major lower limb amputations. Materials and Methods: A prospective, randomized, interventional study was conducted involving 98 patients in Hospital Kuala Lumpur requiring major lower limb amputation due to diabetic complication, tumor and trauma from October 2016 to June 2017. Patients were evenly randomized into two groups; Group A: conventional traction neurectomy (CTN) and Group B: ligation and cauterization neurectomy ( $L C N)$. End point of the study was the outcome of PLP which was assessed clinically at post-operative day 3, 14 and 6-month. Results were analyzed using univariate and multivariate simple logistic regression. Results: There were have 47 and 46 patients in Group A and B respectively. Of these, $70.2 \%$ in Group $A(n=33)$ and $28.3 \%$ in Group B $(n=13)$ end up with PLP $(p<0.001)$. Sixty nine percent of patients who underwent amputation above knee level $(n=20)$ suffered from PLP $(p<0.05)$. Group B patients had higher mean PLP pain score (3.31 and 2.94) but it was not statistically significant. $69.6 \%$ of patients with pre-operative pain $(n=32)$ and $43.5 \%$ with peripheral vascular disease $(n=20)$ developed PLP $(p<0.05)$. Conclusion: LCN has 0.1 chance to develop PLP compared to CTN. Other significant factors in those who developed PLP include levels of amputation, pre-operative pain and peripheral vascular disease. LCN should be considered in primary amputation. 